(․ С.Н. Лысенко ${ }^{1 *}$, М.А. Чечнева' Н.А. Зубкова², А.Н.Тюльпаков

'Московский областной НИИ акушерства и гинекологии, Москва

${ }^{2}$ Национальный медицинский исследовательский центр эндокринологии, Москва

ОБОСНОВАНИЕ. Основой ранней ультразвуковой (УЗ) диагностики диабетической фетопатии (ДФ) у беременных с гестационным сахарным диабетом (ГСД) является своевременное выявление макросомии и особенно асимметричных ее форм. У беременных с ГСД на диете выявление макросомии может являться показанием для начала инсулинотерапии. У беременных с гипергликемией, обусловленной мутацией в гене глюкокиназы (GCK), У3-динамика роста плода помогает предположить генотип плода, а также стратифицировать риски инсулинотерапии.

ЦЕЛЬ. Определить прогностическую значимость выявления макросомии и расчета коэффициентов пропорциональности телосложения плода для диагностики ДФ у беременных с ГСД, в том числе с гипергликемией, обусловленной мутацией в гене GCK.

МЕтОДЫ. Проведена УЗ-фетометрия у 95 беременных с ГСД (в том числе у 22 беременных с впервые выявленной во время беременности гипергликемией, обусловленной мутацией в гене GCK) (основная группа) и у 427 здоровых беременных (контрольная группа). Оценивались предполагаемая масса плода (ПМП), стандартные фетометрические показатели и коэффициенты пропорциональности. Ретроспективный анализ УЗ-предикторов макросомии проводился после оценки массы новорожденного и уточнения признаков ДФ.

РЕзУЛЬтАТЫ. В группе с ГСД у 51 (53,7\%) беременной родились дети с фенотипическими признаками ДФ, в том числе с макросомией - 66,7\% (34 ребенка). Мы выявили статистически значимые различия по ПМП между контрольной группой и группой беременных с ГСД, родивших детей с ДФ, уже с 32-й недели гестации. Коэффициенты пропорциональности (длина бедра/окружность живота (ДБ/ОЖ) и окружность головы/окружность живота (ОГ/ОЖ)), характеризующие формирование асимметричного типа телосложения плода, статистически значимо различались с 34 нед $(\mathrm{P}<0,05)$.

ЗАКЛЮЧЕНИЕ. Наиболее эффективными предиктивными фотометрическими показателями для диагностики макросомии являются размеры живота плода и ПМП >90 перцентиля по сроку гестации. Специфичным признаком ДФ у беременных с ГСД является асимметрия его телосложения. У беременных с мутацией в гене GCK тенденция к макросомии выявлена только при отсутствии мутации у плода, однако инсулинотерапия при наличии аналогичной мутации у плода не приводила к значимому снижению его перцентильных диапазонов.

КЛЮЧЕВЫЕ СЛОВА: гестачионный сахарный диабет; диабетическая фетопатия; макросомия; моногенный диабет

\title{
ULTRASONIC PREDICTORS OF MACROSOMIA IN GESTATIONAL DIABETES MELLITUS
}

(c) Sergey N. Lysenko ${ }^{*}$, Marina A. Chechneva', Fatima F. Burumkulova', Vasiliy A. Petrukhin', Anton E. Panov', Margarita A. Plechanova', Victoria I. Ulyatovskaya', Natalia A. Zubkova², Anatoly N. Tiulpakov²

'Moscow Regional Research Institute of Obstetrics and Gynecology, Moscow, Russia

${ }^{2}$ Endocrinology Research Centre, Moscow, Russia

BACKGROUND: The basis of early ultrasound (US) diagnosis of diabetic fetopathy (DF) in pregnant with gestational diabetes mellitus (GDM) is the forehanded detection of macrosomia, especially its asymmetric forms. In pregnant with GDM on a diet therapy, the detection of macrosomia may be an indication for starting the insulin therapy. In pregnant with hyperglycemia due to mutation in the glucokinase gene (GCK), US fetal growth dynamics helps to assume the fetal genotype, as well as to stratify the risks of insulin therapy.

AIM: To determine the prognostic significance of asymmetric form of macrosomia and the value of the coefficients of proportionality for the diagnosis of DF in pregnant with GDM, including hyperglycemia due to mutation in the GCK gene.

MATERIALS AND METHODS: US fetometry was performed in 95 pregnant with GDM (including 22 pregnant with hyperglycemia caused by mutation in the GCK gene) (main group) and 427 healthy pregnant women (control group). Estimated 
fetal weight, standard fetometric indicators and coefficients of proportionality were evaluated. Retrospective analysis of US predictors of macrosomia was carried out after evaluating the weight of the newborn and clarifying the signs of DF.

RESULTS: In the group with GDM, 51 (53.7\%) pregnant had children with phenotypic symptoms of DF, including macrosomia $-66,7 \%$ (34 children). We found statistically significant differences in fetal weight between the control group and the main group who gave birth to children with DF starting from 32 weeks. The coefficients of proportionality (femur length/ abdominal circumference and the head circumference/abdominal circumference), characterizing the formation of the asymmetric macrosomia were significantly from 34 weeks $(P<0,05)$.

CONCLUSION: The most effective predictive fetometric indicators for the diagnosis of fetal macrosomia are the dimensions of fetal abdomen and fetal weight $>90$ percentile for gestational age. A specific sign of DF in pregnant with GDM is the asymmetric macrosomia. In pregnant with a mutation in the GCK gene, the tendency to macrosomia was revealed only in the absence of a mutation in the fetus, but insulin therapy in the presence of a similar mutation in fetus did not lead to a significant decrease in its percentile ranges.

KEYWORDS: gestational diabetes mellitus, diabetic fetopathy, macrosomia, monogenic diabetes

По данным Международной федерации диабета (IDF), 21,3 млн (16,2\%) детей, рожденных в 2017 г., в период внутриутробного развития подвергались воздействию гипергликемии. По имеющимся оценкам, 86,4\% этих случаев были вызваны гестационным сахарным диабетом (ГСД), 6,2\% - другими типами СД, впервые выявленными до наступления беременности, и 7,4\% - другими его типами (включая СД 1 типа и 2 типа), впервые выявленными во время беременности [1].

Несмотря на «мягкую» гипергликемию, ГСД существенно увеличивает риск неблагоприятных исходов беременности и оказывает долгосрочное негативное воздействие на здоровье матери и ребенка («метаболическое программирование»), включая предрасположенность к ожирению, метаболическому синдрому, СД 2 типа, артериальной гипертензии в более позднем периоде жизни $[2,3,4]$.

В работах C. Crowther и соавт. [5] и M. Landon и соавт. [6] продемонстрировано, что, наряду со своевременным выявлением ГСД, модификация образа жизни и инициация инсулинотерапии приводят к значительному улучшению перинатального исхода и снижению частоты акушерских осложнений.

В исследовании НАРО (Hyperglycemia and Adverse Pregnancy Outcome) выявлена прямая взаимосвязь между увеличением степени гипергликемии у матери с ГСД и такими неблагоприятными исходами беременности, как вес новорожденного >90 перцентиля (П), концентрация С-пептида в пуповинной крови >90П, неонатальная гипогликемия и первое кесарево сечение [7].

Наиболее частым осложнением внутриутробной гипергликемии, независимо от типа СД у матери, является развитие диабетической фетопатии (ДФ). ДФ (код по МКБ-10 Р70-Р74) - это преходящие эндокринные нарушения и нарушения обмена веществ, специфичные для плода и новорожденного, являющиеся следствием эндокринных и метаболических нарушений в организме матери и нарушений эндокринной, а вслед за этим и остальных функций плацентарного комплекса [8].

Самым распространенным признаком ДФ является макросомия (от греч. macrosomia; macro - большой; soma - тело), т.е. увеличение размеров плода выше $90 П$ по нормативным УЗ-таблицам для данного срока гестации [9, 10]. Макросомия плода встречается в 15- 45\% при ГСД, что в 3 раза превышает популяци- онную частоту и напрямую зависит от уровня гликемии у матери [11]. Большие размеры плода значительно увеличивает риск родового травматизма, дистоции плечиков, перелома ключиц, паралича Эрба, экстренного кесарева сечения, послеродового кровотечения или разрывов промежности $[12,13,14]$. Так, S.L. Wood и соавт. [15] отмечают, что мертворождаемость при ГСД (33,7\%о) значительно выше, чем в здоровой популяции $(5,5 \%)$.

Выявление признаков асимметричной макросомии у беременных с ГСД на диете является косвенным свидетельством хронической гипергликемии, требует немедленной коррекции питания, более частого гликемического контроля, а также назначения инсулинотерапии $[7,16]$.

Одним из основных методов диагностики ДФ и макросомии является ультразвуковое исследование (УЗИ), широко применяемое в перинатологии в связи с высокой безопасностью и информативностью. На основании соотношения параметров окружности головки (ОГ) к окружности живота (ОЖ) плода выделяют симметричный (конституциональный - 70\%) и асимметричный (ассоциированный с гипергликемией у матери - 30\%) типы макросомии. Наиболее информативным УЗ-критерием выявления макросомии является динамическое увеличение ОЖ плода >90 П, при этом для асимметричной формы макросомии характерно снижение коэффициентов отношения ОЖ к ОГ плода (ОЖ/ОГ) и отношения длины бедренной кости к ОЖ (ДБ/ОЖ) [12, 13, 17]. Для верификации типа макросомии еще в I триместре уточняются срок беременности [18], личный и семейный анамнез, в дальнейшем анализируют данные УЗИ на протяжении гестации.

По мнению ряда авторов, дополнительное измерение различных УЗ-параметров мягких тканей плода, таких как диаметр бедра, толщина передней брюшной стенки плода и мягких тканей плечиков, в комбинации с измерением ОЖ является наилучшим способом прогнозирования макросомии (чувствительность - 96\%, специфичность - 89\%, точность - 93\%) [19, 20], однако оно требует УЗИ экспертного уровня и не всегда осуществимо в реальной клинической практике.

Важной задачей УЗ-диагностики у пациенток с ГСД является определение предполагаемой массы (ПМП) плода, от которой в значительной мере зависит выбор срока и метода родоразрешения. Прогнозирование 
ПМП основано на использовании уравнений, отражающих математическую зависимость между массой плода и различными его биометрическими параметрами. В настоящее время существует более 100 таких уравнений, которые обладают различной диагностической ценностью и включают в себя от 1 до 6 фетометрических параметров [21]. Для расчета ПМП российские специалисты наиболее часто используют уравнения, предложенные В.Н. Демидовым и соавт. [10, 12], тогда как зарубежные наиболее универсальной и информативной программой считают формулу F. Hadlock и соавт. [22]. Ошибки в определении ПМП провоцируют неоправданное повышение оперативной активности акушеров-гинекологов, при этом частота кесаревых сечений возрастает в 2 раза [23].

Вес ребенка у матерей с гипергликемией, впервые выявленной во время беременности и обусловленной мутацией в гене GCK, прежде всего, зависит от того, наследует плод мутацию или нет. В настоящее время нет возможности рутинно определять генотип плода неинвазивным методом во время беременности, поэтому УЗ-данные позволяют предположить наличие или отсутствие мутации у плода («суррогатный» предиктор), оказывают влияние на выбор терапевтической тактики, могут служить критерием начала инсулинотерапии и оценки ее эффективности $[24,25]$. Так, при прогрессивном увеличении ОЖ плода >75П на фоне диеты можно предположить отсутствие мутации GCK у плода, что требует инициации инсулинотерапии, а при задержке роста плода/уменьшении ОЖ <25П на фоне инсулинотерапии можно предположить наличие у него мутации в гене GCK, что определяет менее «агрессивную» инсулинотерапию, вплоть до полной ее отмены [26, 27, 28]. В работе B.M Shields. и соавт. [29] также выявлено, что дети, унаследовавшие мутацию, имеют меньшую массу плаценты, чем младенцы без мутации.

Н.А. Зубковой и соавт. [30] было показано, что при сравнительном анализе матерей, беременность которых протекала на фоне ГСД, обусловленного мутациями в гене $G C K$, в зависимости от наличия или отсутствия аналогичной мутации у ребенка и получаемой во время беременности терапии, риску развития макросомии подвержены только младенцы, не унаследовавшие материнскую мутацию. Была выявлена тенденция к снижению массо-ростовых показателей у детей с мутацией в гене GCK, чьи матери получали инсулинотерапию во время беременности. Однако снижение показателей веса не было критичным и укладывалось в диапазон нормальных антропометрических показателей для новорожденных.

\section{ЦЕЛЬ}

Определить прогностическую значимость выявления асимметричной формы макросомии и значение коэффициентов пропорциональности телосложения плода для диагностики ДФ у беременных с ГСД, в том числе с гипергликемией, обусловленной мутацией в гене GCK.

\section{МЕТОДЫ}

\section{Дизайн исследования}

Проведено обсервационное одноцентровое ретроспективное выборочное неконтролируемое исследование.

\section{Критерии соответствия}

В исследование были включены здоровые беременные и беременные с ГСД, который был диагностирован согласно рекомендациям Российского национального консенсуса «Гестационный сахарный диабет: диагностика, лечение и послеродовое наблюдение» [16], давшие добровольное согласие на участие в исследовании.

Критерии исключения:

- многоплодная беременность;

- подтвержденные хромосомные аномалии и врожденные пороки развития плода;

- возраст беременной младше 18 лет.

\section{Условия проведения}

Настоящее исследование проводилось в рамках научной работы Московского областного центра «Сахарный диабет и беременность». Клиническое обследование пациенток проведено на базе ГБУЗ МО «Московский областной НИИ акушерства и гинекологии». Набор пациенток проводился в поликлиническом отделении и в акушерских клиниках МОНИИАГ. Диагностика ГСД проводилась согласно рекомендациям Российского национального консенсуса «Гестационный сахарный диабет: диагностика, лечение и послеродовое наблюдение» [16]. Молекулярно-генетический анализ проводился в лаборатории отделения наследственных эндокринопатий ФГБУ «НМИЦ эндокринологии» Минздрава России.

\section{Продолжительность исследования}

Исследование проведено в 2016-2018 гг. Продолжительность периода наблюдения и кратность обследований для каждой пациентки определялась сроком родоразрешения (минимальный срок 30 нед).

\section{Описание медицинского вмешательства}

Суточный мониторинг гликемии проводился беременными амбулаторно с использованием персональных глюкометров и занесением показателей гликемии в дневник самоконтроля. Инсулинотерапия при неэффективности диеты назначалась согласно рекомендациям Российского национального консенсуса «Гестационный сахарный диабет: диагностика, лечение и послеродовое наблюдение» [16].

В основу оценки состояния плода положены УЗ- и допплерометрическое исследования, которые проводились на сроках 11-13; 18-22; 27-30; 34; 37-40 нед беременности при помощи аппарата Medison V-20 компании Samsung.

При УзИ плода оценивались показатели стандартной фетометрии.

1. ОГ, диаметр грудной клетки (ДГ), бипариетальный размер (БПР) головки плода, ОЖ, ДБ.

2. ПМП (рассчитывалась по формуле F. Hadlock [22]: $\Pi \mathrm{M}=\log _{10}(0,064 \times \mathrm{HC})+(0,0424 \times \mathrm{AC})+(0,00061 \times \mathrm{BPD} \times \mathrm{AC})+$ $(0,174 \times F L)-(0,00386 \times A C \times F L)+1,3596$ (г), где: HC - окружность головки плода; AC - окружность животика плода; BPD - бипариетальный размер головки плода; $\mathrm{FL}$ - длина бедренной кости.

3. Коэффициенты пропорциональности тела плода (ОГ/ОЖ, ДБ/ОЖ). Показатели рассчитывались в автоматическом режиме УЗ-аппаратом.

Согласно рекомендациям национального руководства «Неонатология», беременность считалась доношенной на сроке 37-42 нед гестации [31]. Оценка весовых 
показателей новорожденных проводилась сразу после рождения с использованием программы Auxology (Pfizer). Задержка внутриутробного роста (или «малый для гестационного возраста») устанавливалась при снижении массы тела и роста при рождении <10П для гестационного возраста. Диагноз «диабетическая фетопатия» устанавливался на основании увеличения массы тела при рождении выше 2 SD или более $90 П$ в сочетании с признаками диспропорционального развития (увеличение размеров паренхиматозных органов, короткая шея и относительно короткие конечности, маленькая голова, широкий плечевой пояс, толстая шейная складка), утолщения и отека подкожного жирового слоя, признаков неонатальной гипогликемии [31].

Молекулярно-генетическое исследование. Молекулярно-генетический анализ проводился в лаборатории отделения наследственных эндокринопатий ФГБУ «НМИЦ эндокринологии» Минздрава России [32].

\section{Анализ в группах}

В группу окончательного анализа были включены 427 здоровых беременных (контрольная группа), 95 беременных с ГСД (основная группа). Динамическое У3-исследование проводилось всем беременным. После рождения проводилась оценка массы плода по перцентильной шкале для подтверждения прогностической значимости внутриутробной диагностики макросомии и выявления признаков ДФ. В группу ГСД включены также 22 пациентки с впервые выявленной во время беременности гипергликемией, обусловленной мутацией в гене $G C K$, в которой дополнительно проведен анализ фетометрических показателей в зависимости от наличия или отсутствия у ребенка аналогичной мутации.

\section{Методы регистрации исходов}

Данные наблюдения за течением беременности, показатели гликемии, дозы инсулина, результаты молекулярно-генетического исследования, данные УЗ-фетометрии, признаки ДФ, метод лечения (диета или инсулинотерапии), показатели веса новорожденных были занесены в базы данных, сформированные с учетом цели исследования.

\section{Этическая экспертиза}

Данное исследование одобрено локальным этическим комитетом ГБУЗ МО МОНИИАГ (протокол №89 от 30/06/2016 и протокол №88 от 16/06/2016). Информиро- ванное согласие было получено от всех пациенток, в том числе и на обследование их детей.

\section{Статистический анализ}

Статистическая обработка материала проводилась с использованием программ Microsoft Office Excel 2010. Достоверность различий определяли, используя критерий Манна-Уитни. Различия считали достоверными при $\mathrm{p}<0,05$. Проводили дисперсионный анализ КраскеллаУоллиса, а также корреляционный анализ с вычислением коэффициента Спирмена (R) и Гамма (G). Данные представлены как медиана (нижний квартиль; верхний квартиль).

\section{РЕЗУЛЬТАТЫ}

Объекты (участники) исследования

Ретроспективно проведен анализ фетометрических показателей плодов, у которых после рождения неонатологами была подтверждена ДФ, а также плодов от здоровых беременных. В группе с ГСД 39 пациенток $(41,0 \%)$ получали инсулинотерапию. Все беременные с гипергликемией, обусловленной мутацией в гене GCK, получали инсулинотерапию. Медианы возраста беременных в обеих группах были сопоставимы и составили 30 лет [20-43 года]. У 51 (53,7\%) беременной с ГСД родились дети с фенотипическими признаками ДФ, из них с макросомией $66,7 \%$ (34 ребенка). В группе контроля макросомия отмечалась в $12,5 \%$ случаев, что в 2,9 раза реже $(p<0,05)$.

\section{Основные результаты исследования}

ПМП у плодов с ДФ была выше, чем у плодов группы контроля во всех указанных сроках, причем после 32 нед гестации статистически значимо $(p<0,05)$. У плодов без ДФ частота ПМП не отличалась от плодов контрольной группы (табл. 1).

Среди детей, родившихся с ДФ, с макросомией было 34 ребенка (66,7\%), с массой менее $25 П$ - 7 детей (5\%). Среди детей, родившихся без ДФ, с макросомией было 8 новорожденных (18,2\%), с массой тела менее 25П 16 новорожденных (36,4\%). С нормальной массой тела при ДФ родилось 28,3\% новорожденных, без ДФ - 45,4\%.

У плодов с ДФ ОЖ статистически значимо превышала таковую у плодов контрольной группы с 32 нед гестации, при этом во всех сроках ОЖ превышала 89П. У плодов без ДФ ОЖ соответствовала таковой у плодов группы контроля (табл. 2).

Таблица 1. Медиана предполагаемой массы плода (г) по группам (в скобках указаны перцентиль относительно группы контроля и квартильный разброс)

\begin{tabular}{|c|c|c|c|}
\hline \multirow{2}{*}{ Сроки, нед } & \multirow{2}{*}{ Контроль } & \multicolumn{2}{|c|}{ ГСД } \\
\hline & & Без ДФ & ДФ \\
\hline $30-31$ & 1613 [1465-1835] & 1564 (40П) [1530-1598] & 2038 (95П) [1751-2589] \\
\hline $32-33$ & 1963 [1791-2205] & 1739 (17П) [1102-2109] & $2729 *(>99 \Pi)$ [2538-2899] \\
\hline $34-35$ & $2453[2247-2617]$ & $2667 * *(82 \Pi)[1724-3891]$ & $3205^{*}(>99 \Pi)$ [2441-4118] \\
\hline $36-37$ & 3052 [2889-3296] & 3094 (57П) [2380-4619] & $3452 *(85 \Pi)[2470-4243]$ \\
\hline $38-39$ & $3421[3197-3560]$ & 3184 (23П) [2976-3894] & $3747 *(91 \Pi)$ [3162-4327] \\
\hline 40 & 3620 [3401-3928] & 3573 (43П) [3511-3636] & - \\
\hline
\end{tabular}

Примечания: ГСД - гестационный сахарный диабет; ДФ - диабетическая фетопатия; * $-p<0,01$; ** $-p<0,05$. 
Таблица 2. Медиана окружности живота (мм) по группам (в скобках указаны перцентиль относительно группы контроля и квартильный разброс)

\begin{tabular}{|c|c|c|c|}
\hline \multirow{2}{*}{ Срок, нед } & \multirow{2}{*}{ Контроль } & \multicolumn{2}{|c|}{ гСД } \\
\hline & & Без ДФ & ДФ \\
\hline $30-31$ & $261,4[250,1-277,8]$ & 268,4 (57П) [262,4-274,5] & 291,9 (98П) $[284,8-311,1]$ \\
\hline $32-33$ & $279[271,3-292,9]$ & 281,8 (52П) $[224,8-298,4]$ & $325,1 *(>99 \Pi)[314,3-331,5]$ \\
\hline $34-35$ & $302,9[294,3-314,1]$ & $321,5 *(95 \Pi)[272,5-381]$ & $347,9 *(>99 \Pi)[307,0-384,0]$ \\
\hline $36-37$ & $329,9[320,9-339,9]$ & 330,6 (52П) $[298,3-413,1]$ & $350,5 *(89 \Pi)[313,5-377,9]$ \\
\hline $38-39$ & $340,2[330,7-348,0]$ & 327,4 (21П) [320,9-368,8] & $364,9 *(98 \Pi)[337,3-383,1]$ \\
\hline 40 & $352,0[340,6-360,6]$ & 350,1 (48П) [342,6-357,7] & - \\
\hline
\end{tabular}

Примечания: ГСД - гестационный сахарный диабет; ДФ - диабетическая фетопатия; * - p<0,01; ** - p<0,05.

ОЖ у плодов с мутацией в гене GCK в доношенном сроке гестации была статистически значимо ниже, чем ОЖ у плодов без мутации - 60 [33-78] и 92,5 [52,5-97] $(p<0,05)$. У $60 \%$ плодов $(6 / 10)$ без мутации ОЖ в доношенном сроке превышала 90П, однако после рождения макросомия была подтверждена только у одного ребенка (2,22 SD). Снижение ОЖ ниже 25П («здоровый» маловесный ребенок) у плодов с мутацией в гене GCK было выявлено только в $17 \%$ случаев (не было ни одного случая $<10 П)$, тогда как у плодов без мутации - в 10\% (декомпенсированная фетоплацентарная недостаточность (ФПН), хроническая гипоксия плода) (один случай $<10 П)$.

Коэффициенты асимметричности ОГ/ОЖ и ДБ/ОЖ с 34 нед были статистически значимо ниже у плодов с ДФ, чем у плодов без ДФ и группы контроля. У плодов без ДФ после 36 нед эти коэффициенты значимо не отличались от таковых у плодов группы контроля (табл. 3,4$)$.

Следует отметить, что большие размеры живота плода, отражающиеся в виде снижения коэффициента ДБ/ОЖ, выглядят при УЗИ как «относительно короткая» бедренная кость плода.

В группе контроля у плодов с макросомией коэффициент ДБ/ОЖ и ОГ/ОЖ не имел статистически значимых различий с плодами без макросомии (симметричная макросомия). Напротив, в группе ГСД у плодов с макросомией статистически значимые различия с плодами без макросомии появлялись уже с 30 нед $(p<0,01)$. У плодов с ДФ без макросомии, тем не менее, отмечалось статистически значимое снижение коэффициентов пропорциональности плода (асимметричная макросомия). У плодов от матерей с ГСД и отсутствием признаков ДФ по вышеуказанным коэффициентам статистически значимых различий с плодами контрольной группы во всех сроках беременности не выявлено.
Нами была выявлена высокая специфичность обнаружения макросомии (ПМП >90П) у беременных с ГСД с помощью стандартной УЗ-фетометрии. Точность расчета ПМП составляет в 34-35 нед 82,3\%, в 36-37 нед $71,2 \%$, в 38-39 нед - 78,4\%. Прогностическая ценность положительного результата высока до 38 нед гестации (до 72,4\%), а отрицательного результата - во всех указанных сроках (в 34-35 нед - 92,0\%, в 36-37 нед - 70,4\%, в 38-39 нед - 94,6\%). Выявление макросомии при ДФ, несмотря на умеренную чувствительность, высокоспецифично и точно. При обнаружении ПМП менее $90 П$ вероятность Выявления ДФ не более 19,5\%.

Чувствительность и специфичность выявления ОЖ, превышающей 80П, для диагностики ДФ составили в эти же сроки до $80,0 \%$ и 90,0\% соответственно, точность и прогностическая ценность отрицательного результата также были высокими (до 84,0\% и 94,4\% соответственно).

Анализ коэффициентов пропорциональности обнаружил статистически значимые отличия между группой плодов с ДФ и группой контроля по коэффициентам, связанным с размерами живота, при этом чувствительность ОГ/ОЖ в 34-35 нед составляет 75\%, в 36-37 нед - 64,7\%, в 38-39 недель - 71,4\%, специфичность в эти же сроки $90,3 \%, 80 \%$ и $86,4 \%$ соответственно. Точность расчета ОГ/ОЖ составляет в 34-35 нед 84\%, в 36-37 нед - 73\%, в 38-39 нед - 84,3\%. Прогностическая ценность отрицательного результата высока во всех указанных сроках (в 34-35 нед - 84,8\%, в 36-37 нед - 72,7\%, в 38-39 нед 95\%).

Расчет коэффициента асимметричности ДБ/ОЖ максимальной чувствительности достигает в 38-39 нед гестации $(85,7 \%)$ при специфичности 79,5\%, прогностической ценности отрицательного результата $97 \%$ и точности $84,3 \%$.

Таблица 3. Медиана окружности головки к окружности живота по группам (в скобках указаны перцентиль относительно группы контроля и квартильный интервал)

\begin{tabular}{|c|c|c|c|}
\hline \multirow{2}{*}{ Срок, нед } & \multirow{2}{*}{ Контроль } & \multicolumn{2}{|c|}{ гСД } \\
\hline & & Без ДФ & ДФ \\
\hline $30-31$ & $1,08[1,04-1,10]$ & $1,01(3 П)[1,012-1,017]$ & $0,98(<1 П)[0,96-0,99]$ \\
\hline $32-33$ & $1,04[1,01-1,09]$ & 0,97 (ЗП) $[0,97-1,04]$ & 0,99 (7П) $[0,96-1,00]$ \\
\hline $34-35$ & $1,01[0,99-1,06]$ & $0,98 * *(10 \Pi)[0,96-1,02]$ & $0,94 *(1 \Pi)[0,89-0,97]$ \\
\hline $36-37$ & $0,98[0,95-1,00]$ & 0,98 (50П) $[0,94-1,02]$ & $0,93 *(11 \Pi)[0,90-0,96]$ \\
\hline $38-39$ & $0,98[0,95-1,02]$ & 0,94 (7П) $[0,93-0,96]$ & $0,91 *(3 \Pi)[0,90-0,94]$ \\
\hline 40 & $0,95[0,91-0,98]$ & 0,93 (37П) $[0,91-0,94]$ & - \\
\hline
\end{tabular}

Примечания: ГСД - гестационный сахарный диабет; ДФ - диабетическая фетопатия; * $-p<0,01 .{ }^{* *}-p<0,05$. 
Таблица 4. Медиана длины бедренной кости к окружности живота по группам (в скобках указаны перцентиль относительно группы контроля и квартильный интервал)

\begin{tabular}{|c|c|c|c|}
\hline \multirow{2}{*}{ Срок, нед } & \multirow{2}{*}{ Контроль } & \multicolumn{2}{|c|}{ гСД } \\
\hline & & Без ДФ & ДФ \\
\hline $30-31$ & $22,3[21,9-23,2]$ & 21,04 (10П) $[20,5-21,5]$ & 21,47 (14П) $[20,7-21,7]$ \\
\hline $32-33$ & $22,4[21,6-23,1]$ & 20,76 (<1П) $[20,6-22,3]$ & $19,5(<1 \Pi)[19,4-21,0]$ \\
\hline $34-35$ & $22,2[21,7-23,0]$ & $21,41 *(18 \Pi)[21,04-21,75]$ & $20,44 *(<1 \Pi)[19,4-21,7]$ \\
\hline $36-37$ & $21,9[21,3-22,4]$ & 21,65 (33П) $[20,85-22,60]$ & $21,11 *(18 \Pi)[19,8-21,6]$ \\
\hline $38-39$ & $22,3[21,7-23,0]$ & 22,77 (68ח) $[21,47-23,44]$ & $21,13 *(10 \Pi)[20,8-21,5]$ \\
\hline 40 & $22,3[21,4-23,0]$ & 22,11 (45П) $[21,91-22,31]$ & - \\
\hline
\end{tabular}

Примечания: ГСД - гестационный сахарный диабет; ДФ - диабетическая фетопатия; * - p<0,01.

Нежелательные явления

В данном исследовании нами не были отмечены нежелательные явления.

\section{ОБСУЖДЕНИЕ}

В настоящее время отмечается возобновление интереса врачебного мирового сообщества к совершенствованию методов прогнозирования ПМП [33], что в значительной степени определяет сроки и методы родоразрешения и крайне актуально для беременных с СД. В нашей работе были использованы стандартные показатели фетометрии, доступные для измерения на уровне первичного приема женской консультации и рассчитываемые У3-аппаратом в автоматическом режиме.

Макросомия плода при ГСД встречается в 25-42\% случаев [7, 12, 13], что соответствует полученным данным (35,8\%). Наиболее рано макросомия выявлялась в 18-19 нед гестации, однако статистически значимые различия между контрольной группой и группой беременных с ГСД появились только с 34 нед. У3-предикторы макросомии имели высокую точность (до 82,3\%), чувствительность (до 90,0\%) и специфичность (до 79,5\%).

Макросомия в обеих группах в первую очередь была обусловлена увеличением размеров живота плода, что требовало определения точного срока гестации [18], измерения особенностей его телосложения и уточнения симметричности. По данным Hackmon R. и соавт. [34], макросомия может выявляться уже с 11-14 нед гестации, при этом Salomon L. и соавт. [35] ранним предиктором макросомии считают увеличение копчико-теменного размера (КТР) в эти сроки. Если при первом УзИ КТР эмбриона $\geq 7$ дней от срока последней менструации, риск рождения плода более 4500 г составляет 59\% [36].

Асимметричность телосложения плода является специфичным признаком ДФ. Многие авторы $[13,37,38]$ указывают, что диспропорция телосложения плода при ГСД выявляется только с 28-29 нед беременности, однако, по нашим данным, ее можно зафиксировать уже с 16-17 нед (в случаях раннего выявления ГСД или пропущенного манифестного СД) [17]. Следует отметить, что для выявления макросомии в эти сроки необходимо ориентироваться не только на сроки гестации, но и на перцентильную оценку фетометрических показателей $[22,39]$.

В группе ГСД при формировании ДФ плоды без макросомии и даже с ПМП <25П имели такую же форму асимметрии, как и плоды с макросомией, несмотря на маленькую массу тела. Напротив, для формирования симметричной макросомии (конституциональной), выявляемой у плодов от матерей без СД, было характерно равномерное увеличение фетометрических показателей с отсутствием различий по коэффициентам ОГ/ОЖ и ДБ/ОЖ. По данным литературы, соотношение ДБ/ОЖ и ОГ/ОЖ уменьшается при асимметричной макросомии с чувствительностью 80\% и точностью 82\% [40], что сопоставимо с полученными нами данными.

При гипергликемии, обусловленной мутацией в гене $G C K$, развитие макросомии было характерно только для плодов, не имеющих аналогичной мутации, их фетометрические параметры выходили за границы 75П в начале III триместра беременности, особенно при позднем начале или некорректном подборе инсулинотерапии. Такие плоды чаще имели тенденцию к макросомии с формированием ДФ. У плодов, которые унаследовали GCK мутацию и матери которых получали инсулинотерапию во время беременности, были выявлены тенденция к снижению фетометрических параметров и отсутствие макросомии $[25,26]$.

Снижение показателей веса при рождении у обследованных нами беременных с гипергликемией, обусловленной мутацией в гене GCK, не было критичным и укладывалось в диапазон нормальных антропометрических показателей для новорожденных. Это согласуется с данными G.B. Spyer и соавт. [25], которым не удалось обнаружить влияния инсулинотерапии матери на вес ребенка при рождении. Тем не менее авторы, наряду с другими, придерживаются мнения о необходимости определения генотипа матери и плода при выборе оптимальной тактики ведения таких пациентов [41].

Следует отметить, что при оценке темпов роста плода у матерей с мутацией в гене GCK необходимо учитывать показатели допплерометрии (объемный кровоток в артерии пуповины, отражающий перфузию между плацентой и плодом), так как причиной формирования маловесного плода может быть не только наличие мутации, но и ФПН или присоединение преэклампсии. При ФПН, как основной причине патологической маловесности плода, определяющим фактором является снижение объемного кровотока в артерии пуповины [42].

\section{ЗАКЛЮЧЕНИЕ}

Таким образом, у беременных с ГСД перцентильная оценка УЗ-фетометрических данных должна прово- 
диться начиная с момента постановки диагноза и до родоразрешения. Диагностика ДФ должна строиться на выявлении макросомии (ОЖ>90П) и уточнении ее формы на основании коэффициентов пропорциональности телосложения плода.

Асимметричность телосложения при макросомии за счет увеличения размеров живота может являться скрининговым признаком, причем, чем более выражена асимметричность, тем тяжелее ДФ. Кроме этого, она служит дополнительным критерием инициации инсулинотерапии у беременных с ГСД при неэффективности диеты.

Наряду с фенотипическими (увеличение толщины подкожной клетчатки шеи, живота, головы, увеличение ширины лопатки, увеличение буккального индекса) и висцеральными (гепато-спленомегалия, кардиомиопатия, увеличение толщины поджелудочной железы) признаками, макросомия плода является критерием диагностики ДФ.

Золотым стандартом при лечении беременных с мутацией в гене GCK и неизвестным генотипом у плода по-прежнему является инсулинотерапия, в то время как
УЗ-параметры роста плода (ПМП, ОЖ) начиная со II триместра могут являться «суррогатными» предикторами генотипа плода и, как следствие, определять оптимальные целевые уровни компенсации гипергликемии у матери и выбор метода лечения (диета или инсулинотерапия).

\section{ДОПОЛНИТЕЛЬНАЯ ИНФОРМАЦИЯ}

Финансирование исследования. Молекулярно-генетическое исследование выполнено за счет гранта Российского научного фонда (проект №16-15-10408).

Конфликт интересов. Авторы декларируют отсутствие явных и потенциальных конфликтов интересов, связанных с публикацией настоящей статьи.

Участие авторов. Лысенко С.Н., Бурумкулова Ф.Ф.: концепция и дизайн исследования, анализ полученных данных, написание текста; Чечнева М.А., Панов А.Е., Петрухин В.А., Улятовская В.И., Плеханова М.А.: сбор материала, анализ полученных данных; Тюльпаков А.Н., Зубкова Н.А.: проведение молекулярно-генетического исследования. Все авторы внесли значимый вклад в проведение исследования и подготовку статьи, прочли и одобрили финальную версию перед публикацией.

\section{СПИСОК ЛИТЕРАТУРЫ | REFERENCES}

1. International Diabetes Federation. IDF Diabetes Atlas. 8th ed. Brussels: IDF; 2017

2. Patel S, Fraser A, Davey Smith G, et al. Associations of gestational diabetes, existing diabetes, and glycosuria with offspring obesity and cardiometabolic outcomes. Diabetes Care. 2012;35(1):63-71. doi: https://doi.org/10.2337/dc11-1633

3. Katzmarzyk PT, Barreira TV, Broyles ST, et al. The International Study of Childhood Obesity, Lifestyle and the Environment (ISCOLE): design and methods. BMC Public Health. 2013;13:900. doi: https://doi.org/10.1186/1471-2458-13-900

4. Huopio $\mathrm{H}$, Hakkarainen $\mathrm{H}$, Paakkonen $\mathrm{M}$, et al. Long-term changes in glucose metabolism after gestational diabetes: a double cohort study. BMC Pregnancy Childbirth. 2014;14:296. doi: https://doi.org/10.1186/1471-2393-14-296

5. Crowther CA, Hiller JE, Moss JR, et al. Effect of treatment of gestational diabetes mellitus on pregnancy outcomes. N Engl J Med. 2005;352(24):2477-2486. doi: https://doi.org/10.1056/NEJMoa042973

6. Landon MB, Spong CY, Thom E, et al. A multicenter, randomized trial of treatment for mild gestational diabetes. N Engl J Med. 2009;361(14):1339-1348. doi: https://doi.org/10.1056/NEJMoa0902430

7. Hod M, Kapur A, Sacks DA, et al. The International Federation of Gynecology and Obstetrics (FIGO) Initiative on gestational diabetes mellitus: A pragmatic guide for diagnosis, management, and care. Int J Gynaecol Obstet. 2015;131:S173-S211. doi: https://doi.org/10.1016/s0020-7292(15)30033-3

8. Committee on Fetus and Newborn, Adamkin DH. Postnatal glucose homeostasis in late-preterm and term infants. Pediatrics. 2011;127(3):575-579. doi: https://doi.org/10.1542/peds.2010-3851

9. Медведев М.В., Юдина Е.В. Задержка внутриутробного развития плода. — М.: РАВУЗДПГ; 1998. [Medvedev MV, Yudina EV. Zaderzhka vnutriutrobnogo razvitiya ploda. Moscow: 1998. (In Russ.)]

10. Демидов В.Н. Розенфельд Б.Е. Возможности прогнозирования массы и роста плода при однократном и многократных исследованиях в III триместре при физиологически развивающейся беременности // SonoAce International. - 2000. №6. [Demidov VN, Rosenfeld BE. Vozmozhnosti prognozirovaniya massy i rosta ploda pri odnokratnom i mnogokratnykh issledovaniyakh v III trimestre pri fiziologicheski razvivayushcheysya beremennosti. SonoAce International. 2000;(6). (In Russ.)]

11. Kc K, Shakya S, Zhang H. Gestational diabetes mellitus and macrosomia: a literature review. Ann Nutr Metab. 2015;66 Suppl 2:14-20. doi: https://doi.org/10.1159/000371628
12. Ордынский В.Ф., Макаров О.В., Постникова Н.А. Значение допплерометрической оценки артериального кровотока в системе мать-плацента-плод у беременных с сахарным диабетом // Ультразвуковая и функциональная диагностика. 2003. - №2. - C. 22-34. [Ordynsky VF, Makarov OV, Postnikova NA. The Importance of the Ultrasound Dopplerographic Estimation of the Arterial Blood Flow in the System Mother-Placenta-Fetus in the Pregnants with Diabetes Mellitus. Ultrasound \& functional diagnostics. 2003;(2):22-34. (In Russ.)]

13. Ордынский В.Ф., Макаров О.В. Сахарный диабет и беременность. Пренатальная ультразвуковая диагностика: руководство для врачей. — М.: Видар-M; 2010. [Ordynsky VF, Makarov OV. Sakharnyy diabet i beremennost'. Prenatal'naya ul'trazvukovaya diagnostika. A guide for doctors. Moscow: Vidar-M; 2010. (In Russ.)]

14. Vitoratos $\mathrm{N}$, Vrachnis $\mathrm{N}$, Valsamakis $\mathrm{G}$, et al. Perinatal mortality in diabetic pregnancy. Ann N Y Acad Sci. 2010;1205:94-98. doi: https://doi.org/10.1111/j.1749-6632.2010.05670.x

15. Wood SL, Jick H, Sauve R. The risk of stillbirth in pregnancies before and after the onset of diabetes. Diabet Med. 2003;20(9):703-707. doi: https://doi.org/10.1046/j.1464-5491.2003.01015.x

16. Дедов И.И., Краснопольский В.И., Сухих Г.Т. Российский национальный консенсус: «Гестационный сахарный диабет: диагностика, лечение, послеродовое наблюдение» // Сахарный диабет. - 2012. - Т. 15. - №4. - C. 4-10. [Dedov II, Krasnopol'skiy VI, Sukhikh GT. Russian National Consensus Statement on gestational diabetes: diagnostics, treatment and postnatal care. Diabetes mellitus. 2012;15(4):4-10. (In Russ.)] doi: https://doi.org/10.14341/2072-0351-5531

17. Антенатальная ультразвуковая диагностика диабетической фетопатии и макросомии (информачионно-методическое письмо). / Под ред. Краснопольского В.И. — М.; 2013. [Krasnopolskiy VI, editor. Antenatal'naya ul'trazvukovaya diagnostika diabeticheskoy fetopatii i makrosomii (informatsionno-metodicheskoe pis'mo). Moscow; 2013. (In Russ.)]

18. Salomon LJ, Alfirevic Z, Bilardo CM, et al. ISUOG practice guidelines: performance of first-trimester fetal ultrasound scan. Ultrasound Obstet Gynecol. 2013:41(1):102-113. doi: https://doi.org/10.1002/uog.12342

19. Lee W. OC106a: Fractional limb volume: a new soft-tissue parameter for the evaluation of fetal size and growth. Ultrasound Obstet Gynecol. 2003;22(S1):30-30. doi: https://doi.org/10.1002/uog.318

20. Lee W, Deter RL, McNie B, et al. Individualized growth assessment of fetal soft tissue using fractional thigh volume. Ultrasound Obstet Gynecol. 2004;24(7):766-774. doi: https://doi.org/10.1002/uog.1779 
21. Campbell S. Fetal macrosomia: a problem in need of a policy. Ultrasound Obstet Gynecol. 2014;43(1):3-10. doi: https://doi.org/10.1002/uog.13268

22. Hadlock FP, Harrist RB, Sharman RS, et al. Estimation of fetal weight with the use of head, body, and femur measurements-A prospective study. Am J Obstet Gynecol. 1985;151(3):333-337. doi: https://doi.org/10.1016/0002-9378(85)90298-4

23. Баева И.Ю. Клиническая ценность дородовой диагностики крупного плода по данным ультразвуковых исследований. // Журнал акушерства и женских болезней. - 2014. - Т. 63. - №3. - С. 12-20. [Bayeva IY. Clinical significance of prenatal diagnosis of macrosomia by ultrasound. Journal of obstetrics and women's diseases. 2014;63(3);12-20. (In Russ.)] doi: https://doi.org/10.17816/JOWD63312-20

24. Spyer G, Hattersley AT, Sykes JE, et al. Influence of maternal and fetal glucokinase mutations in gestational diabetes. Am J Obstet Gynecol. 2001;185(1):240-241. doi: https://doi.org/10.1067/mob.2001.113127

25. Spyer G, Macleod KM, Shepherd M, et al. Pregnancy outcome in patients with raised blood glucose due to a heterozygous glucokinase gene mutation. Diabet Med. 2009;26(1):14-18 doi: https://doi.org/10.1111/j.1464-5491.2008.02622.x

26. Colom C, Corcoy R. Maturity onset diabetes of the young and pregnancy. Best Pract Res Clin Endocrinol Metab. 2010;24(4):605-615. doi: https://doi.org/10.1016/j.beem.2010.05.008

27. Chakera AJ, Spyer G, Vincent N, et al. The $0.1 \%$ of the population with glucokinase monogenic diabetes can be recognized by clinical characteristics in pregnancy: the Atlantic Diabetes in Pregnancy cohort. Diabetes Care. 2014;37(5):1230-1236. doi: https://doi.org/10.2337/dc13-2248

28. Chakera AJ, Steele AM, Gloyn AL, et al. Recognition and Management of Individuals With Hyperglycemia Because of a Heterozygous Glucokinase Mutation. Diabetes Care. 2015;38(7):1383-1392. doi: https://doi.org/10.2337/dc14-2769

29. Shields BM, Hicks S, Shepherd MH, et al. Maturity-onset diabetes of the young (MODY): how many cases are we missing? Diabetologia. 2010;53(12):2504-2508. doi: https://doi.org/10.1007/s00125-010-1799-4

30. Зубкова Н.А., Бурумкулова Ф.Ф., Петрухин В.А., и др. Весо-ростовые показатели детей, рожденных от матерей с гестационным сахарным диабетом, обусловленным мутациями в гене глюкокиназы // Сахарный диабет. — 2018. — Т. 21. — №2. — С. 9298. [Zubkova NA, Burumkulova FF, Petrukhin VA, et al. Birth weight and length in offsprings of mothers with gestational diabetes mellitus due to mutations in GCK gene. Diabetes Mellitus. 2018;21 (2):92-98 (In Russ.)] doi: https://doi.org/10.14341/DM9429

31. Володин Н.Н. Неонатология. Начиональное руководство. - М.; 2008. [Volodin NN. Neonatologiya. National guidelines. Moscow; 2008. (In Russ.)]

32. Зубкова Н.А., Гиоева О.А., Тихонович Ю.В., и др. Персонализация коррекции нарушений углеводного обмена с учетом генотипа у пациентов с сахарным диабетом типа MODY, обусловленного мутациями в генах GCK, HNF1A, HNF4A // World Journal of Personalized Medicine. - 2017. - T. 1. — №1. — C. 40-48. [Zubkova NA, Gioeva OA, Tikhonovich YV, et al. Genotype-based personalized correction of glycemic control in patients with MODY due to mutations in GCK, HNF1A AND HNF4A genes. World Journal of Personalized Medicine. 2017;1 (1):40-48. (In Russ.)] doi: https://doi.org/10.14341/wjpm9298

33. Merialdi M, Widmer M, Gulmezoglu AM, et al. WHO multicentre study for the development of growth standards from fetal life to childhood: the fetal component. BMC Pregnancy Childbirth. 2014;14:157. doi: https://doi.org/10.1186/1471-2393-14-157

34. Hackmon R, Le Scale KB, Horani J, et al. Is severe macrosomia manifested at 11-14 weeks of gestation? Ultrasound Obstet Gynecol. 2008;32(6):740-743. doi: https://doi.org/10.1002/uog.5310

35. Salomon LJ, Hourrier $S$, Fanchin R, et al. Is first-trimester crown-rump length associated with birthweight? BJOG. 2011;118(10):1223-1228. doi: https://doi.org/10.1111/j.1471-0528.2011.03009.x

36. Thorsell M, Kaijser M, Almstrom H, Andolf E. Large fetal size in early pregnancy associated with macrosomia. Ultrasound Obstet Gynecol. 2010;35(4):390-394. doi: https://doi.org/10.1002/uog.7529

37. Black RS, Gillmer MDG. Diabetes in pregnancy. Obstet Gynaecol. 2003;5(3):143-148. doi: https://doi.org/10.1576/toag.5.3.143

38. Schaefer-Graf UM, Wendt L, Sacks DA, et al. How Many Sonograms Are Needed to Reliably Predict the Absence of Fetal Overgrowth in Gestational Diabetes Mellitus Pregnancies? Diabetes Care. 2010;34(1):39-43. doi: https://doi.org/10.2337/dc10-0415

39. Приказ Минздрава России N 457 от 28 декабря 2000 «О совершенствовании пренатальной диагностики в профилактике наследственных и врожденных заболеваний у детей». [Order of the Ministry of Health of Russia N 457 of 28 Dec 2000 «O sovershenstvovanii prenatal'noy diagnostiki v profilaktike nasledstvennykh i vrozhdennykh zabolevaniy u detey». (In Russ.)]

40. Ancuta E, Ancuta C, Sofroni D, et al. P14.05: Assessment of abdominal circumference among 29-33 gestational weeks adding a three hour oral glucose tolerance test can predict macrosomia. Ultrasound Obstet Gynecol. 2011;38(S1):215-215. doi: https://doi.org/10.1002/uog.9783

41. Chakera AJ, Carleton VL, Ellard S, et al. Antenatal diagnosis of fetal genotype determines if maternal hyperglycemia due to a glucokinase mutation requires treatment. Diabetes Care. 2012;35(9):1832-1834. doi: https://doi.org/10.2337/dc12-0151

42. Ермакова Л.Б., Лысенко С.Н., Чечнева М.А., и др. Особенности гемодинамики в артерии пуповины у беременных с сахарным диабетом и у здоровых беременных // Российский вестник акушера-гинеколога. - 2016. - Т. 16. — №4. - С. 54-60. [Ermakova LB, Lysenko SN, Chechneva MA, et al. Umbilical artery hemodynamic features in diabetic and healthy pregnant women. Rossiiskii vestnik akushera-ginekologa. 2016;16(4):54-60 (In Russ.)] doi: https://doi.org/10.17116/rosakush201616454-60

\section{ИНФОРМАЦИЯ ОБ АВТОРАХ [AUTHORS INFO]}

*Лысенко Сергей Николаевич, к.M.Н., С.н.с. [Sergey N. Lysenko, MD, PhD, research associate]; адрес: Россия, 101000 Москва, ул. Покровка, д. 22a [address: 22a Pokrovka street, 101000 Moscow, Russian Federation]; ORCID: https://orcid.org/0000-0003-3280-2799; eLibrary SPIN: 3442-6268; e-mail: serzwer@mail.ru

Чечнева Марина Александровна, д.м.н. [Marina A. Chechneva, MD, PhD]; ORCID: https://orcid.org/0000-0001-7066-3166; eLibrary SPIN: 2292-6321; e-mail: marina-chechneva@yandex.ru Зубкова Наталья Анатольевна, к.M.н., С.н.с. [Natalia A. Zubkova, MD, PhD, research associate]; ORCID: https://orcid.org/0000-0002-1346-7545; eLibrary SPIN: 5064-9992; e-mail: zunata2006@yandex.ru Тюльпаков Анатолий Николаевич, д.м.н. [Anatoliy N. Tyulpakov, MD, PhD]; ORCID: https://orcid.org/0000-0001-8500-4841; eLibrary SPIN: 8396-1798; e-mail: ant@endocrincentr.ru Бурумкулова Фатима Фархадовна, д.м.н., в.н.с. [Fatima F. Burumkulova, MD, PhD, leader research associate]; ORCID: https://orcid.org/0000-0001-9943-0964; eLibrary SPIN: 6592-7736; e-mail: fatima-burumkulova@yandex.ru Петрухин Василий Алексеевич, д.м.Н., профессор [Vasily A. Petrukhin, MD, PhD, Professor]; ORCID: https://orcid.org/0000-0003-0460-3047; eLibrary SPIN: 9236-6783; e-mail: petruhin271058@mail.ru

Улятовская Виктория Ивановна, врач акушер-гинеколог [Victoria I. Ulyatovskaya, MD]; ORCID: https://orcid.org/0000-0002-0930-3476; elibrary SPIN 2988-9072; e-mail: 1akmoniiag@mail.ru

Панов Антон Евгеньевич, н.с. [Anton E. Panov, MD, research associate];

ORCID: https://orcid.org/0000-0002-1299-7456; eLibrary SPIN: 9328-7938; e-mail: drpanov82@gmail.com

Плеханова Маргарита Александровна, врач-эндокринолог [Margarita A. Plechanova, MD]; ORCID: http://orcid.org/0000-0002-5322-1021; eLibrary SPIN: 8860-9060; e-mail: margarita_kr@list.ru 


\section{ЦИТИРОВАТЬ:}

Лысенко С.Н., Чечнева М.А., Бурумкулова Ф.Ф., Петрухин В.А., Улятовская В.И., Панов А.Е., Плеханова М.А., Зубкова Н.А., Тюльпаков А.Н. Ультразвуковые предикторы формирования макросомии при гестационном сахарном диабете // Сахарный диабет. - 2019. — Т. 22. — №4. — С. 358-366. doi: 10.14341/DM10109

\section{TO CITE THIS ARTICLE:}

Lysenko SN, Chechneva MA, Burumkulova FF, Petrukhin VA, Ulyatovskaya VI, Panov AE, Plechanova MA, Zubkova NA, TyuIpakov AN. Ultrasonic predictors of macrosomia in gestational diabetes mellitus. Diabetes Mellitus. 2019;22(4):358-366. doi: 10.14341/DM10109 